Alexis Boisson, Cession et licence en droit d'auteur.

Alexis Boisson, Cession et licence en droit d'auteur, Legicom, 2014, $\mathrm{n}^{\circ}$ 53, Propriété intellectuelle : notions cadres et mécanismes essentiels, pp. 59-68.

\title{
Cession et licence en droit d'auteur
}

« Si dans un passé ancien, la nature exacte de ces contrats a pu être discutée, tout un chacun s'accorde aujourd'hui, depuis la remarquable thèse de $M$. Burst sur la licence de brevet, pour tenir la licence pour une location dans le champ des propriétés intellectuelles. De même que la cession n'est pas analysée autrement qu'en termes de vente. Cela valant bien sûr pour toutes les propriétés intellectuelles, du droit d'auteur à la marque $\gg^{1}$. Les débats relatifs à la nature des contrats de la propriété intellectuelle devraient-ils être soustraits des manuels de droit de la propriété intellectuelle et venir enrichir d'un nouveau chapitre les manuels d'histoire du droit ? Rien n'est moins sûr car - davantage que ceux du juge certes - les travaux de la doctrine spécialisée comme «civiliste » s'intéressant au sujet sont récents et nombreux ${ }^{2}$.

\section{Cession et licence en propriété industrielle}

Cession et licence cohabitent en propriété industrielle. La première est assimilée à la vente, ou plus généralement aux contrats emportant un effet réel ; la seconde est rattachée au louage de choses et parfois au prêt à usage en cas de gratuité. Cette summa divisio directement issue du droit civil est nettement affirmée en matière de brevet, où I'article L. 613-8 du Code de la propriété intellectuelle (CPI) nomme, sans vraiment les régir, les deux formules : une «transmission 》 (en fait une cession) et une «concession de licence d'exploitation, exclusive ou non exclusive ». Des termes similaires sont employés en droit des marques (art. L. 714-1) et en droit des dessins et modèles (art. L. 513-2). Si cette distinction est ancienne ${ }^{3}$, c'est à Roubier que l'on doit une définition de référence de la licence : « contrat par lequel le titulaire d'un monopole d'exploitation concède à une personne en tout ou partie la jouissance de son droit d'exploitation ${ }^{4}$. L'auteur démontre la «véritable parenté » entre la licence et le louage de choses, tandis que la cession est rapprochée de la vente. A vrai dire, l'analyse a tant marqué les esprits que I'on tend à oublier la prudence de l'analogie proposée par Roubier, qui concluait à une licence, contrat sui generis. Quant à la cession, elle ne pouvait

\footnotetext{
${ }_{1}^{1}$ M. Vivant (dir.), Les créations immatérielles et le droit, Ellipses, 1997, p. 25 (référence faite à J.-J. Burst, Breveté et licencié. Leurs rapports juridiques dans le contrat de licence, Librairies techniques, 1970).

2 N. Blanc, Les contrats du droit d'auteur à l'épreuve de la distinction des contrats nommés et innommés, Dalloz, 2010 ; S. Raimond, La qualification du contrat d'auteur, Litec, IRPI, 2009 ; A. Boisson, La licence de droit d'auteur, LexisNexis, 2013. - La décennie précédente est tout aussi prolifique : J. Raynard, Droit d'auteur et conflits de lois. Essai sur la nature juridique du droit d'auteur, Litec, 1990 ; E. Tardieu-Guigues, La licence de marque, Thèse, Montpellier, 1991. - Les principales thèses récentes en droit des biens traitent de la question, dernièrement: M. Jaoul, La notion de fruits, Etude de droit privé, thèse, Montpellier I, 2014.

3 V. E. Pouillet, Traité théorique et pratique de la propriété littéraire et artistique et du droit de représentation, $3^{e}$ éd., par Maillard et Claro, Eds Marchal et Billard, 1908, nº 385.

${ }_{4}^{4}$ P. Roubier, Licences et exclusivités, Ann. dt commercial, 1936, p. 289 ; Le droit de la propriété industrielle, TII, Sirey, 1954, p. 286.
} 
produire un effet réel, à l'image des «droits intellectuels », dits « de clientèle », qu'il se refusait à rattacher à la propriété privée. Retenons cependant la force de I'opposition de deux formules concurrentes, qui seront purement et simplement assimilées à la vente et à la location, à la faveur d'une pleine admission des propriétés industrielles au rang de véritables propriétés. Aujourd'hui, dans cette branche de la propriété intellectuelle, les débats de qualifications portent non plus tant sur les contrats eux-mêmes que sur leurs nouvelles modalités, voire leur « esprit $»^{5}$. En définitive, nous nous rangeons à ce constat introductif : pour ce qui relève de la propriété industrielle, le débat sur les catégories contractuelles semble globalement apaisé.

\section{Cession et licence en droit d'auteur}

En matière de droit d'auteur, en revanche, la question reste vivement discutée. Le débat s'articule autour de la prééminence du contrat de « cession ». Plusieurs fois nommée par le livre $1^{\mathrm{er}}$ du CPI, celle-ci est la clé de voute du droit contractuel d'auteur. Le droit spécial de ces contrats (art. L. 132-1 et Ss. CPI) fait trôner une cession au sein des contrats d'édition et de production audiovisuelle. Les dispositions générales (art. L. 131-1 CPI) s'en tiennent au terme de cession (ou de transmission), de sorte que tout contrat d'auteur est désigné - directement ou indirectement par référence à ce droit « commun »- comme étant une cession. On aurait pu espérer qu'une certaine variété naisse de I'« autorisation » siégeant dans les contrats de représentation (art. L. 132-18). Or, d'une part, cette « autorisation » échappe aux définitions habituellement reçues pour ce terme en droit privé ${ }^{\text {. }} \mathrm{D}^{\prime}$ autre part, c'est bien à un droit des « cessions » que la soumettent les dispositions générales du CPI. En somme, le droit d'auteur, droit de propriété pourtant ${ }^{7}$, laisse apparaître quant à ses contrats un paysage bien peu familier au civiliste. Un camaïeu dans lequel les formules les plus variées : définitives ou temporaires, exclusives ou non-exclusives, ne seraient que les nuances d'une même couleur, recouvrant la palette des usages que I'on peut faire d'une œuvre. Il était donc prévisible qu'une importante doctrine ${ }^{8}$ voie dans la cession la seule formule concevable. Sans s'encombrer de distinctions, les juges usent dans leur grande majorité du terme « cession », celui de « licence » étant repris sans plus de discernement lorsqu'il s'agit d'appliquer le droit européen de la concurrence ${ }^{9}$. Fidèles à la lettre de la loi, certains chercheront à faire tenir cette diversité toute entière dans ce terme unique : «Plutôt que de focaliser l'attention sur l'introuvable frontière entre cession et licence, il nous paraît plus fécond (...) de mettre l'accent sur l'idée que le monopole d'auteur se démembre à volonté ${ }^{10}$. La cession qui,

\footnotetext{
${ }^{5}$ V. Ch. Caron, L'efficacité des licences dites « FRAND », CCE, juil. 2013, étude 12 ; Ph. StoffelMunck, in Les nouveaux usages du brevet, Colloque de la Chaire Innovation \& brevets, Univ. AixMarseille, 26-27 sept. 2014, à paraître. Leur caractère « irrévocable » pose question cependant.

${ }^{6}$ V. B. Thullier, L'autorisation. Etude de droit privé, LGDJ, 1996, évoquant l'acte unilatéral.

7 Cons. const., déc. du 27 juil. 2006, n²006-540 DC, RTD civ. 2006, p. 791, obs. Th. Revet.

8 Hier, par ex. A. Françon, La liberté contractuelle dans le domaine du droit d'auteur, D. 1976, Chron., p. 55. Aujourd'hui : Ph. Gaudrat, Les modèles d'exploitation du droit d'auteur, RTD com. 2009, p. 323 ; A. et H.-J. Lucas, A. Lucas-Schloetter, Traité de la propriété littéraire et artistique, $4^{\mathrm{e}}$ éd., Litec, $2012, n^{\circ} 532$.

9 Notons également les Lignes directrices concernant l'application de l'article 101 du TFUE à des catégories d'accords de transfert de technologie (JOUE 28 mars 2014, $\mathrm{N}^{\circ} \mathrm{C} 89, \mathrm{p} .3$ ) évoquant la «licence de droit d'auteur » et le « preneur ».

${ }^{10}$ A. et H.-J. Lucas, A. Lucas-Schloetter, op. cit., terme repris à J. Dabin, Le droit subjectif, Dalloz, 1952, p. 190.
} 
dès lors, n'est plus tout à fait une vente, serait elle-même susceptible de tous les degrés: en intensité puisqu'elle serait exclusive ou non-exclusive; mais également dans le temps. Cette doctrine est méritoire en ce qu'elle cherche à donner un sens aux mots du législateur. Elle semble en revanche illusoire, car en légiférant la «cession» plutôt que la licence, notre législateur n'a fait que reprendre une terminologie antérieure et dépourvue de consistance. Desbois avait déjà noté que le législateur, cherchant à instaurer un régime contractuel protecteur de l'auteur, avait négligé la question des qualifications ${ }^{11}$. Plus d'un siècle avant la loi du 11 mars 1957 qui la consacra, on percevait déjà le caractère générique de la « cession » en droit d'auteur : «Les droits attachés au privilège d'auteur sont cessibles. Ils peuvent être transmis à titre gratuit ou onéreux, par louage, vente, prêt, mandat, donation entre vifs ou testamentaire, et par tout autre mode $\gg^{12}$. En d'autres termes, dire que l'œuvre est cessible signifierait lato sensu qu'elle peut être l'objet de contrats.

Ce malentendu levé, il revient à présent au juriste de découvrir, sous les terminologies, la réalité juridique préexistante. Bien qu'il y ait toujours une place pour l'innommé, le cadre des contrats spéciaux du Code civil donnera un sens à ces notions. Une vente, une location : chacun s'accorde sur ces termes. Certains de leurs éléments tenus pour essentiels seront communs aux deux formules et n'auront donc pas d'effet sur leurs qualifications (I); d'autres éléments au contraire, nous donneront les clés pour mieux les distinguer (II).

\section{$\underline{\text { I - Cession et licence : éléments communs }}$}

Ce qui est commun est essentiel, mais ne peut servir à distinguer. Cession et licence ont pour points communs de porter, directement pour l'une, indirectement pour l'autre, sur une œuvre de l'esprit (A) ; elles sont aussi soumises à un dispositif impératif conçu pour protéger l'auteur $(B)$.

\section{A-Cession et licence : des contrats sur une chose}

\section{1 - Des contrats portant sur l'œuvre}

Cession et licence sont des contrats portant sur une chose. Cette chose est dépourvue de corps, il s'agit de l'œuvre de l'esprit protégée par un régime de propriété spécial, le droit d'auteur. A ce titre, le contrat de «licence d'utilisation » de logiciel, qui a la prétention d'accorder un droit d'utilisation n'est en rien une licence de droit d'auteur; il n'est pas davantage une cession. Ce contrat a pour objet de lever un obstacle physique (hier un emballage, aujourd'hui une activation en ligne) afin de laisser au client l'usage d'un outil, par ailleurs protégé par le droit d'auteur. Or, si l'exploitation d'un logiciel met en jeu le droit d'auteur, son

\footnotetext{
11 H. Desbois, Le droit d'auteur en France, 3e éd., Dalloz, 1978, p. 608.

12 A.-C. Renouard, Traité des droits d'auteurs, dans la littérature les sciences et les arts, t. 2,

J. Renouard et Cie Libraires, 1839, p. 278.
} 
utilisation à titre d'outil, quant à elle, ne relève d'aucune propriété13. La licence d'utilisation organise l'accès à un "service capitalisé » ${ }^{14}$ qu'est l'usage d'un logiciel. Sans même entrer dans la distinction cession/licence, la «licence » d'utilisation échoue à son entrée dans le cercle des contrats de propriété intellectuelle d'une part, des contrats sur les choses d'autre part. En revanche, dès lors que le contrat dépassera cet objet, en autorisant des actes de reproduction ou de représentation au-delà de ce que la loi permet à tous, nous serons en présence d'un contrat d'exploitation qu'il restera à qualifier.

\section{2 - Des contrats portant sur les seules utilités patrimoniales de l'œuvre}

Les droits moraux interrogent les catégories contractuelles sur deux niveaux de réflexion bien distincts.

Selon un premier point de vue, l'inaliénabilité des droits moraux du vivant de l'auteur (art. L. 121-1 et ss. CPI) s'opposerait à toute cession de l'œuvre. Surmonter pareil obstacle suppose de cerner l'objet qui sera effectivement vendu ou loué. Si le rattachement symbolique des droits moraux aux droits de la personnalité est un vaste sujet de controverses, en revanche on s'accorde sur leur traduction en termes d'effets juridiques. En cette circonstance, l'objet d'un contrat en droit d'auteur portera exclusivement sur ce qui, dans les utilités de l'œuvre, est disponible. Ce qui est retranché à la cession opérée par l'auteur sera en pareils termes retranché de la licence. Cette réalité s'impose quelle que soit la conception retenue pour les droits moraux : un ersatz de droit de la personnalité, une composante pleine et entière d'une propriété débarrassée de sa connotation purement patrimoniale ${ }^{15}$ ou encore une simple série de dérogations au régime normal de la propriété ${ }^{16}$. Les droits moraux sont, par principe, extérieurs à l'objet du contrat. Ils ne viennent donc pas en troubler la qualification.

D'un autre point de vue, on doit bien admettre que les droits moraux vont influer sur ce à quoi s'engagent les parties. Les droits de divulgation, de retrait et de repentir, le droit de l'auteur au respect de son nom (paternité) et de son œuvre, troubleront à différents titres la jouissance du cessionnaire, comme celle du licencié : en suspendant les effets du contrat, en conduisant à sa résolution, ou encore en permettant à l'auteur d'opérer un contrôle sur les actes d'exploitation du contractant. Mais toutes ces dérogations portent atteinte à des obligations communes à toutes sortes de contrats sur les choses et non à la qualification de ces catégories constituant les contrats spéciaux. Un droit de retrait n'est pas un «terme » qui transformerait toute cession en licence, pas plus que le réméré ne transforme la vente en bail ${ }^{17}$. Les droits moraux restent donc, à tous points de vue, neutres en matière de qualifications contractuelles.

\footnotetext{
13 Depuis la loi du 3 juil. 1985 jusqu'à celle du 10 mai 1994, I'art. L. 122-6 CPI disposait que I'utilisation du logiciel pouvait être interdite ou autorisée par son propriétaire. Rappr. I'accès pour lecture à un livre numérique.

14 Déjà : H. Croze et Y. Bismuth, Le contrat dit de licence de logiciel, JCP E, 1986, II, 14659. - Sur ce débat, v. notre thèse, La licence de droit d'auteur, LexisNexis, 2013, $\mathrm{n}^{\circ} 62$ et ss.

15 F. Zenati-Castaing et Th. Revet, Les biens, 3e éd., PUF, 2008, n73.

16 Ce qui constitue la thèse de $\mathrm{J}$. Raynard, op. cit.

17 Pour une analyse de ces droits en droits potestatifs, v. notre thèse précitée, n 252 et ss.
} 


\section{B - Cession et licence : un régime impératif partagé}

Une série de dispositions vient inhiber une liberté contractuelle jugée par trop dangereuse pour l'auteur. Ce droit impératif se manifeste de deux manières. D'une part un droit « commun » qui s'impose à tout contrat mettant en scène un auteur et son œuvre ${ }^{18}$ (caractère en principe proportionnel de la rémunération, prohibition des cessions globales d'œuvres futures). D'autre part, un droit tout aussi impératif, mais « spécial », car relié à certains contrats d'exploitation régis par la loi (contrat d'édition, de production, de représentation...). On constate ici une complémentarité entre une formule simple, par laquelle l'auteur autorise un tiers à user de son œuvre (cession ou licence) et une série de contrats d'exploitation complexes car complétant cette autorisation d'obligations de faire allant très au-delà de celles d'un simple acheteur ou d'un locataire (composition, promotion, distribution, etc.). Ces dispositifs interrogent à la fois la possibilité même d'une licence (1) et la distinction entre cession et licence (2).

\section{1 - Droit impératif et possibilité de la licence}

Ce droit impératif est tenu pour un obstacle à la reconnaissance d'une licence aux côtés d'une cession ${ }^{19}$. Le droit des contrats d'auteur étant un droit des «cessions», on craint qu'une licence différenciée de la cession permette de contourner ce dispositif impératif obtenu de haute lutte, ramenant le contrat d'auteur à un droit commun du bail, réputé fruste et libéral. Cette idée doit être combattue. Le formalisme contractuel, principal instrument de protection dessiné pour l'auteur, illustrera le propos. La notion regroupe des exigences a priori distinctes, touchant souvent au fond: l'exigence d'un contrat constaté par écrit pour les contrats d'édition, de représentation, de production audiovisuelle (art. L. 131-2 du CPI) ; la règle selon laquelle « La transmission des droits de l'auteur est subordonnée à la condition que chacun des droits cédés fasse l'objet d'une mention distincte dans l'acte de cession et que le domaine d'exploitation des droits cédés soit délimité quant à son étendue et à sa destination, quant au lieu et quant à la durée » (art. L. 131-3 du CPI). De ces articles naît une apparente contradiction : le premier assigne à l'exigence d'un écrit (ad probationem ${ }^{20}$ ) un domaine très restreint, limité à quelques contrats importants, le second pose (ad validitatem ${ }^{21}$ ) quant au contenu de cet écrit une exigence des plus générales. Tout l'enjeu est alors de savoir si le formalisme de la « cession » échappe à la licence.

Cette crainte repose, croyons-nous, sur une lecture inexacte des textes. En effet, si le législateur n'a pas voulu qualifier les contrats dont il a prévu le régime, le texte qui impose un formalisme à la « cession » ne différencie pas non plus cession et licence. Autrement dit, le raisonnement selon lequel le formalisme étant attaché à la cession, les contrats de licence lui échapperaient est doublement faux. Il est d'abord hâtif de considérer les contrats d'exploitation listés comme effectivement organisés sur une cession et non sur une licence. Ensuite, contrairement à une

\footnotetext{
18 Ce qui exclut le cessionnaire ou le licencié, v. Cass. civ. 1 $1^{\text {re }}, 13$ oct. 1993, Bull. I, nº 284.

19 S. Joly, La création artistique et I'ordre public, Thèse, Montpellier, 1999, n 266 ; Ph. Gaudrat, article précité.

20 Cass. civ. 28 mai 1963, JCP G, 1963, II, 13347, obs. Ph. Malaurie.

${ }^{21}$ Cass. civ. $1^{\text {re }}, 23$ janv. 2001, CCE avril 2001, comm. 34, Ch. Caron.
} 
interprétation dominante, le formalisme contractuel n'est pas plus attaché à la cession qu'à la licence, mais à certains contrats d'exploitation nommés et à la présence de l'auteur en tant que partie. La question de l'étendue exacte du formalisme n'est pas encore résolue, mais la Cour de cassation tend à se plier à une lecture regroupée des deux textes cités (qui n'en faisaient qu'un avant la codification de 1992) ${ }^{22}$. L'un donne une liste de contrats à établir par écrit, mettant ainsi en jeu des qualifications, quand l'autre prescrit à sa suite un régime en détaillant les thèmes à spécifier dans les contrats préalablement listés. De la sorte, un contrat de cette liste, organisé sur la base d'une licence et non d'une cession, se voit soumis au formalisme, tandis que la cession pure et simple d'une œuvre commandée ou créée par un salarié ne devrait pas l'être. Si l'on reprochera aux textes de ne pas protéger uniformément les auteurs, ce manque n'est pas imputable à la distinction entre cessions et licences, mais à l'articulation entre ces formules simples et leur utilisation dans un contrat organisant une exploitation. La licence n'est pas une notion neuve permettant de s'affranchir d'un système existant ; elle traduit juridiquement des situations existantes réunies à tort sous la bannière de la « cession ».* [V. mise à jour in fine]

Enfin, pareille hostilité à la licence manque sa cible. Rattachée à la location, la licence aligne la durée de la prestation de l'auteur sur la durée prévisible d'exploitation de l'œuvre. Elle rompt avec la tradition qui confère à l'exploitant une propriété aussi perpétuelle qu'inutile, qu'un formalisme contractuel se borne à enregistrer. Certes, l'idée selon laquelle le choix d'une qualification civiliste protégera mieux la partie faible qu'un dispositif impératif, complexe et ambigu, peut déconcerter.

\section{2 - Droit impératif et distinction entre cession et licence}

D'autres dispositions impératives interfèrent plus directement sur le contenu obligationnel. Pourraient-elles perturber notre distinction ? Nous prendrons pour illustration la question du prix ${ }^{23}$. Celle-ci est multiple, car le juriste considérera son existence même, sa nature, sa détermination ou encore sa « durée ».

En premier lieu, le prix peut ne pas exister. La cession, causée par une intention libérale, se détachera de la vente et deviendra donation ; la licence s'émancipera quant à elle de la location et deviendra prêt à usage. La licence étant un bail, le terme devrait être remplacé. La pratique imposera malgré tout le terme de licence (les «licences libres » sont le plus fréquemment gratuites, mais elles sont aussi définitives ce qui met en doute leur qualification en licence). Ensuite, quid d'un contrat sans prix mais « intéressé », telle l'autorisation gracieuse de l'auteur en quête de notoriété ? Voilà bien de quoi relancer le débat entre les conceptions « objectives » ou « subjectives » de la gratuité ; mais rien n'est ici spécifique à la propriété intellectuelle ${ }^{24}$.

Vient ensuite la question de la nature du prix : monétaire ou autre. Celle-ci ne sert cependant pas à opposer la cession et la licence, mais à distinguer ces formules des différents types d'apports en société en nature : en propriété et en usufruit ou

22 Cass. civ. $1^{\text {re }}, 21$ nov. 2006, CCE janv. 2007, p. 28, obs. Ch. Caron ; Cass. civ. $1^{\text {re }}, 2$ juil. 2014, n०13-24359.

23 Sur l'obligation d'exploitation, v. notre thèse précitée, $n^{\circ} 224$ et ss.

${ }^{24}$ Ibid. n 196. 
en jouissance. Ainsi, I'« apport » des œuvres d'un auteur à la SACEM n'en est pas vraiment un car il n'est pas rétribué par une part sociale, laquelle est liée au paiement d'un droit d'entrée (v. art. 2, 6 et 7 des Statuts).

Quant au mode de détermination du prix, son caractère proportionnel de principe ou exceptionnellement forfaitaire (art. L. 131-4 CPI) n'est pas censé affecter notre distinction. Sans entrer ici dans ce vaste débat, admettons que le prix de la vente et du bail ont vocation à être déterminés ou déterminables. Comment expliquer alors que le prix du contrat d'édition, modèle de «cession » selon la doctrine dominante, soit largement indéterminable à la conclusion du contrat ? Il dépend en effet d'un acte unilatéral de l'éditeur fixant le prix unique de vente des exemplaires, assiette du pourcentage intéressant l'auteur. Doit-on y voir une dérogation au principe de détermination du prix, ou encore un argument soulignant I'originalité de la cession et de la licence ? Il n'en est rien : si le prix du contrat d'édition n'a pas à être déterminé, c'est parce que ce contrat ne se résume ni à une cession ni à une licence. Les contrats d'exploitation du CPI et leurs avatars innommés sont des contrats complexes comportant une autorisation (licence ou cession) ainsi qu'une série d'obligations de faire à charge de l'exploitant, réalisant un service, ce qui les rend irréductibles aux qualifications de vente ou de bail. Si le prix des contrats du CPI n'a pas à être déterminé, ou déterminable, ce n'est donc pas par exception à la règle de détermination du prix, mais parce que ces contrats échappent au domaine d'application de cette règle ${ }^{25}$. Rappelons qu'en droit commun, si la règle de détermination a pu céder en cas de bail, les contrats en question mêlaient bail et entreprise dans un contrat mixte ${ }^{26}$. Quand bien même, cession et licence à l'état pur se rencontrent parfois, de fait, sans prix déterminé ou déterminable : I'auteur, hors commande d'œuvres de nature technique, n'a en général que faire d'un prix déterminé, lui qui souhaite être associé au succès, par nature imprévisible, de son œuvre.

Enfin, la question de la détermination du prix ne doit pas être confondue avec celle de la « durée du prix », plus exactement avec la question de son paiement, étalé dans le temps ou au contraire au comptant. Le prix forfaitaire sera souvent payé en une fois tandis que le prix proportionnel sera très vraisemblablement payé en plusieurs fois. Mais l'inverse est tout aussi concevable. Ces différentes modalités du prix n'affectent pas les qualifications : le prix n'est que la réciproque d'une obligation caractéristique. Or, les modalités de ce qui n'est pas caractéristique ne sauraient disqualifier.

25 Sur le sujet, v. notre thèse précitée, $n^{\circ} 713$.

26 Un des arrêts d'Ass. plén. du $1^{\text {er }}$ déc. 1995 (n॰ 91-15999) concernait un contrat de «locationentretien », au visa des articles 1709 et 1710 du Code civil. 


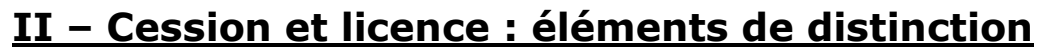

Par l'effet réel, caractéristique de la cession, le cessionnaire jouira directement de l'œuvre transférée. Par l'effet personnel, caractéristique de la licence, le licencié est autorisé à jouir de la chose par l'intermédiaire du donneur de licence. Le niveau d'abstraction de ces catégories fait leur force autant que leur limite : les actes d'exploitation autorisés à l'un ou à l'autre pourront être identiques quant à leur nature. A ces catégories, il faut donc des critères. La présence ou l'absence d'un effet de retour sera le critère de notre distinction $(A)$, quand d'autres éléments $n$ 'influeront que sur la seule qualification de la cession (B).

\section{$\underline{\text { A - L'effet de retour, critère de distinction de la cession et de la licence }}$}

\section{1 - Effet de retour : notion et manifestations}

L'esprit de retour désigne la volonté des parties de conférer une certaine durée à l'autorisation accordée. Rappelons que le bail dure « un certain temps » (art. 1709 C. civ.). Cet esprit se manifeste par un effet de retour : l'extinction du droit personnel d'usage concédé au licencié (I'œuvre n'ayant jamais quitté le patrimoine de l'auteur). Au contraire, la cession ne s'inscrit pas de la même manière dans le temps. La clause usuelle indiquant que l'œuvre est « cédée pour la durée du droit d'auteur », n'a pas pour but d'inscrire la cession dans la durée, mais bien au contraire de marquer son caractère instantané et définitif (ce qui est « définitif » l'est relativement à la durée d'existence de la chose ${ }^{27}$ ). Si la cession échappe alors au grief de perpétuité, c'est parce que «l'auteur aliène sa chose, pas sa personne ${ }^{28}$. La cession de droit d'auteur ne devrait donc plus être analysée en contrat successif. Les obligations de paiement et d'exploitation pourront être successives, mais l'obligation de l'auteur - obligation de donner - caractéristique du contrat, se sublimera en un trait de temps. En somme, l'exigence légale de fixer la durée des «cessions» n'est pas le signe qu'elles puissent être temporaires, mais qu'un choix est ouvert entre licence et cession.

Comment se manifeste cet esprit de retour ? Par une clause de durée donc, mais également par un nombre limité d'actes d'exploitation autorisés : d'exemplaires, de téléchargements... Le contrat dit $d^{\prime}$ « achat de droits de diffusion télévisuelle » est bien mal nommé, lui dont le terme extinctif associe une durée et un nombre donné de diffusions. Le contrat d'édition, tenu pour l'archétype du contrat de cession, connaît déjà un dispositif, certes peu usité, de résiliation unilatérale (art. L. 132-17). Cette circonstance nous incite à voir dans la « cession » figurant dans ce contrat une licence. La récente réforme destinée à faire vivre ce contrat à l'ère du numérique facilite désormais considérablement l'exercice de ce droit par I'auteur. Elle efface, non pas de la lettre de la loi, mais bien en substance, ce lien entre cession et contrat d'édition ${ }^{29}$. En revanche, le contrat de production

\footnotetext{
27 L'œuvre entre dans le domaine public 70 ans post mortem auctoris, marquant la fin des droits patrimoniaux.

28 P.-Y. Gautier, Propriété littéraire et artistique, 8e éd., PUF, 2012, n 458.

29 Ord. n० 2014-1348 du 12 nov. 2014 (v. le nouvel art. L. 132-17-4 CPI). Sur ce texte, en projet, v. A. Boisson, De l'éditeur propriétaire à l'éditeur locataire : la discrète révolution du contrat
} 
audiovisuelle, dont le régime impératif est neutre quant à la durée, pourra contenir aussi bien une licence qu'une cession.

Une vision «arithmétique » de la durée du contrat serait cependant bien frustrante, car seul importe l'esprit de retour. Cette donnée devrait s'apprécier à I'aune de la possibilité d'une nouvelle exploitation à l'issue du contrat. Devrait être qualifié de cession le contrat par lequel l'usage de l'œuvre, retrouvé par le donneur de «licence», ne lui permettrait cependant pas d'accomplir une nouvelle exploitation. Ce qui est temporaire ou définitif pourrait donc être relatif et dépendre du type d'œuvre et des moyens requis pour en entreprendre l'exploitation. A l'inverse d'autres baux d'exploitation construits sur un objectif de stabilité, le contractant protégé est ici le bailleur et non le locataire. Son intérêt se trouvera, généralement, dans la brièveté du droit concédé.

\section{2 - Effet de retour et concurrence des qualifications}

Ce critère de durée étant affirmé, se joue ensuite une concurrence de qualifications. La durée est essentielle au bail, sans pour autant lui être exclusive. La jouissance temporaire d'une chose n'est pas nécessairement locative. Ainsi, dans l'écosystème des contrats de propriété intellectuelle, un contrat à effet réel pourrait occuper la même «niche écologique» qu'une licence : fiducie, constitution ou cession d'usufruit, cessions temporaires, conditionnelles ou encore « faux bail » produisant des effets réels... Le principe de cette concurrence étant accepté, il convient de passer à la confrontation. Alors que l'usufruit ne saurait être concédé à une personne morale au-delà de 30 ans, une hypothétique vente temporaire supposerait un double transfert difficilement praticable. Quant à la vente sous condition résolutoire (dont le réméré), son effet destructeur du contrat serait tout à fait étranger à la « normalité » attendue d'un terme contractuel. Enfin, la fiducie, traditionnellement rapprochée des contrats du droit d'auteur ${ }^{30}$, ne semble pas plus adaptée. La licence se borne à la mise à disposition, contre un prix, d'une œuvre que le licencié exploitera pour son propre compte. Il en percevra les fruits en locataire « raisonnable » sans le secours de cette essence fiduciaire. Si un (bon) éditeur veille activement aux intérêts notamment moraux de l'auteur, on ne saurait affirmer pour autant que tout licencié agisse dans l'intérêt et pour le compte de l'auteur, en dépit d'une obligation de rendre compte, somme toute banale. L'existence d'un patrimoine fiduciaire et son sort en cas de dettes devraient clore le débat (art. 2025), mais à cette divergence d'esprit viennent s'ajouter une série d'incompatibilités de régime. A moins d'imaginer quelque fiducie innommée propre au droit d'auteur, admettons qu'un contrat d'auteur prétendant à la qualification fiduciaire devrait encourir la nullité à maints égards, notamment : caractère solennel (art. 2012 C. civ.) et qualité du fiduciaire (art. 2015 C. civ.). Ces diverses formules concurrentes à la licence doivent être écartées à la fois pour des incompatibilités de régime qu'en raison de l'inutile complexité qu'elles ajoutent

d'édition, RDLC, avril 2014, n¹14, p. 78. - Citons également la loi n²012-287 du $1^{\text {er }}$ mars 2012 relative à l'exploitation numérique des livres indisponibles du XXe siècle (art. L.134-1 et Ss. CPI), permettant aux éditeurs d'obtenir des « autorisations »d'exploitation (exclusives de 10 ans ou nonexclusives de 5 ans) : des licences qui ne disent pas leur nom ! V. J.-M. Bruguière, P. Deprez, Les sept nouveaux chapitres du droit du livre numérique, Légicom n51, 2014/1, p 12.

30 P.-Y. Gautier, op. cit., n० 473. 
au débat. De cette concurrence entre contrats dotés d'un effet de retour, la licence, bail d'exploitation, ressort largement gagnante.

\section{B - Exclusivité et défense du droit, éléments secondaires de qualification.}

L'exclusivité, aux puissants enjeux, décrit les effets d'un contrat plutôt que sa nature juridique. En effet, son origine peut être « réelle » ou « personnelle ». Elle ne saurait donc être un critère de distinction entre cession et licence. Pourtant, l'absence d'exclusivité est souvent reliée à la licence. Tel est le cas des diverses « licences » de logiciel, des licences libres portant sur des logiciels ou toute autre œuvre, celles encore permettant de puiser dans les banques d'images. C'est (outre leur origine américaine) la non-exclusivité consubstantielle à ces opérations définitives qui conduit à préférer le terme de licence. Or, cette conclusion est bien hâtive : ce n'est pas parce que la licence peut être non-exclusive que tout contrat non-exclusif est une licence. Un retour au droit civil doit clarifier la situation. L'exclusivité y apparaît en argument de disqualification davantage qu'en critère de qualification : si l'exclusivité n'est pas le propre de la licence, la non-exclusivité en revanche écarte la cession.

Tout d'abord, la licence peut ne pas être exclusive, ce qui ne perturbe pas sa qualification en bail. Le bail pourrait être non-exclusif : aux parties de convenir de l'intensité ou du partage de leur jouissance, pourvu qu'elle soit paisible ${ }^{31}$. Mais c'est plus vraisemblablement la nature de l'œuvre qui dicte la solution. En effet, la jouissance des choses corporelles se fait au moyen d'une appréhension par les sens tendant à une exclusivité, plus factuelle que juridique. Les choses incorporelles, quant à elles, sont dotées d'ubiquité. Leur jouissance consiste en des actes de représentation et de reproduction qui peuvent se réaliser sans exclusivité. La convention viendra se substituer aux contours matériels de la chose corporelle, qui supposait en principe une emprise solitaire. L'exclusivité, qui n'est alors qu'un élément accidentel du contrat de licence ${ }^{32}$, n'est donc pas un critère de distinction entre licence et cession. Elle n'en demeure pas moins un outil important de qualification : son absence permet de disqualifier la « cession » définitive et non exclusive en formule innommée dans laquelle le droit cédé se retrouve à l'identique dans le patrimoine du cédant et du cessionnaire. S'agissant d'un droit de propriété ou d'usufruit, il peut être partagé, mais il demeure nécessairement exclusif.

Ainsi, l'exclusivité ne qualifie ni ne disqualifie la licence. En revanche, le droit, en corrigeant les effets de certaines exclusivités, encourage ponctuellement le recours à la licence. Le CPI n'a ici qu'un rôle marginal, quand le droit public de l'audiovisuel, par exemple, vient règlementer durées et exclusivités ${ }^{33}$. L'exclusivité est également un facteur d'application du droit de la concurrence. Ce droit, procédant d'une logique autre que la propriété intellectuelle, touche indirectement le contenu du contrat, et donc sa qualification. Aussi a-t-il été appliqué pour sanctionner la pratique de contrats exclusifs à durée excessive au regard des nécessités de

\footnotetext{
31 La Cour de cassation tend à exclure le bail non exclusif, cependant cette carence apparaît parmi un faisceau d'indices qui remettent en cause la jouissance. V. Cass. civ. 3e 11 janv. 2006, Defrénois, $n^{\circ} 9, n^{\circ} 38385$, note E. Savaux.

32 Cass. com. 14 nov. 2006, n 04-16338 (licence de marque).

33 V. I'art. L. 132-19 sur le contrat de représentation. - V. notre thèse précitée, n 528 et ss.
} 
l'exploitation ${ }^{34}$. La Commission européenne a instillé un caractère temporaire à l'«apport» aux sociétés de gestion collective, en sanctionnant les clauses subordonnant le retrait d'un membre à une «condition de transaction non équitable $\gg^{35}$.

Ces développements n'épuisent en rien le débat. En témoigne la question de la titularité de l'action en contrefaçon, pleine d'analogies avec les discussions relatives à l'exclusivité. Comparée à une action en revendication depuis Josserand, sa titularité est fréquemment présentée comme preuve du partage entre cession en licence, ou plus fréquemment entre exclusivité et non exclusivité. Or, en droit d'auteur, la situation apparaît plus confuse qu'en propriété industrielle : la titularité de l'action en contrefaçon échappe non seulement à la distinction entre cession et licence, mais aussi à la distinction entre exclusivité et non-exclusivité. Au mieux, on ne conçoit pas un véritable cessionnaire privé de la défense de son droit. Dans les autres cas (licence exclusive ou non), la titularité de l'action résulte d'une habilitation spéciale trouvant sa source dans les usages et les contrats plus que dans la loi. Dans le silence de la loi, l'éditeur en est investi ; d'autres, également licenciés exclusifs, en sont privés ${ }^{36}$. La situation de l'auteur ayant «apporté » à titre exclusif ses œuvres à la SACEM est, quant à elle, source d'incertitudes ${ }^{37}$. On ne peut qu'espérer qu'une consécration légale des licences au côté des cessions conduise le législateur à trancher cette question et bien d'autres encore.

\section{Alexis Boisson, Maître de conférences en droit privé Université de Montpellier Centre du Droit de I'Entreprise}

* [Mise à jour au 10/03/2021]

Article L131-2 du CPI :

« Les contrats de représentation, d'édition et de production audiovisuelle définis au présent titre doivent être constatés par écrit. Il en est de même des autorisations gratuites d'exécution.

Les contrats par lesquels sont transmis des droits d'auteur doivent être constatés par écrit. [Ajouté par LOI n²016-925 du 7 juillet 2016]

Dans tous les autres cas, les dispositions des articles 1359 à 1362 du code civil sont applicables. »

Sur les conséquences encore incertaines de ce nouvel alinéa sur le sens du texte, v. par ex. Alexis Boisson. «L'article L131-2 du Code de la Propriété intellectuelle ». Colloque des JUSPI, le 4 mars 2021, sur le thème « Le Code de la propriété intellectuelle en dix articles », Dir. Amélie Favreau, Mar 2021, Grenoble, France. 〈hal-03161344)

Notre intervention : https://lesjuspi.wordpress.com/les-videos-des-contributeurs/

34 Cass. com., 26 nov. 2003, Bull. IV, n० 178, CCE 2004, comm. 20, note G. Decocq.

35 CJCE 21 mars 1974, aff. 127/73, Rec. p. 313 ; CJCE, 25 oct. 1979, RIDA, janv. 1980, p. 136. Déc. Commission GEMA I, 2 juin 1971, JOCE L. 134 du 20 juin 1971, p. 15 et GEMA II, 6 juil. 1972, JOCE L. 94 du 8 avril 1982, p. 12.

36 Concernant certains droits voisins, la titularité de l'action du licencié exclusif est admise (art. L. 331-1 CPI). Ce n'est pas le cas en droit d'auteur (Cass. civ. $1^{\text {re }}, 22$ janv. 2009, RTD com. 2009, p. 307, obs. F. Pollaud-Dulian).

37 Admettant l'exercice de l'action par l'auteur : Cass. civ. $1^{\text {re }}, 24$ févr. 1998, obs. A. Françon, RTD com. 1998, p. 592. Amorçant un revirement, sous réserve de la « carence de cette société »: Cass. $1^{\text {re }}$ civ., 13 nov. 2014, n¹3-22410. 